\title{
Agent and Radio Frequency Identification Based Architecture for Supermarket Information System
}

\author{
Hasan Al-Sakran \\ Department of MIS, College of Business Administration, King Saud University, Riyadh, Saudi Arabia
}

Received 2013-02-26, Revised 2013-05-28; Accepted 2013-06-01

\begin{abstract}
In recent years the acceptance of Radio Frequency Identification (RFID) technology in business environments has been increasing rapidly due to its competitive business value. Adopting a suitable RFIDbased information system has become increasingly important for supermarkets. However, most supermarkets still use conventional barcode-based systems to manage their information processes, which are consistently reported as one of the most unenthusiastic aspects of supermarket shopping for both customers and management. We propose an RFID agent-based architecture that adopts intelligent agent technology with an RFID based applications. RFID provides capability to uniquely identify an object within a supermarket area, while agents are able to establish a channel of communication which can be used to facilitate communications between a RFID device and supermarket back-end system. The proposed framework includes a design of intelligent mobile shopping cart equipped with both RFID and agent technologies. As a result of using the proposed RFID agent based architecture, the customer shopping experience will be improved due to ease of retrieving of the detailed information on items and quick checkout by scanning all items at once, thus eliminating queues. From supermarket management point of view the proposed architecture will reduce the cost of operation e.g., decreasing cost of goods sold which comes in the form of labor efficiency in areas of checkout operation, inventory management and alerting the supermarket management when a certain product is running out of stock and needs to be restocked.
\end{abstract}

Keywords: RFID, NFC, Agent, Supermarket, Intelligent Shopping Cart

\section{INTRODUCTION}

During the last decade the commercial use of RFID has been growing rapidly all over the world. Furthermore, it is projected that the RFID market will reach an estimated US\$18.7 billion by the year 2017 (GIAI, 2012).

Everywhere retailers are increasingly embedding RFID technology into their supermarket products in order to improve the customers' shopping experience, customer support and develop new services for customers.

RFID is a technology that uses radio waves to track, capture, identify and transfer data efficiently and without human intervention. RFID-based system gathers data about a certain object without touching it or seeing it stag and forwards the information to a host computer. The data on the tag includea pointer to the central database within an RFID system. RFID-Readers are able to establish a channel of communication, read the tags and trace the movement of these objects within the coverage area.

RFID is a promising technology which can improve operational efficiency specially a considerable amount of reduction in transaction costs. Tag detection does not require human intervention therefore reduces employment costs and eliminates human errors during data collection. Due to its flexibility and business efficiency, the RFID technology has been widely adopted in a wide range of applications such as supply chain management and inventory, libraries, equipment and parts maintenance, vehicle identification, tracking people, access control, reliable car tracking, manufacturing line control, automated reading and receipt of goods at end sale points, e-passport and much more (Owunwanne and Goel, 2010). Implementation of RFID based applications has become an objective of many 
organizations, partially as a result of the decision made by Wal-Mart, the world's biggest retailer, to implement the RFID technology to monitor flow of pallets and packaging in its supply chain and ask their top 100 vendors deploy RFID. The US Department of Defense, Proctor and Gamble and the European retailer Metro Group require their larger suppliers to implement RFID on every box and pallet shipped to them.

The grocery industry is a prime candidate for RFID implementation. On-hand shelf inventory system in the supermarket will be linked to the store's information system, thus maintaining real time product information and automatic inventory tracking to keeping the correct inventory levels (Luo and Wang, 2012). Using RFID technology in supermarkets can also provide detailed information on customer purchase behavior (Takai and Yada, 2010).

Currently most of the supermarkets use a barcodebased system whereby an item is assigned a serial number printed on the barcode label attached to an item and the item related information is stored in the database of the back-end system. To perform inventory control, someone has to scan the barcode label of each item and compare them with existing inventory list. This is a lengthy and error prone process; as a result it's done less frequently and hence often is not up-todate. The capabilities of the barcode technology are limited in term of functionalities that businesses require (Bendavid et al., 2006). RFID technology offers a solution to the above mentioned problem.

RFID has many beneficial features over barcode such as:

- RFID doesn't require line-of-sight access to read as barcode required

- RFID reading range of is much greater distances than that of barcode

- $\quad$ RFID tags can be read simultaneously while barcode is reading one by one

- $\quad$ RFID tags can store large amounts of data of goods than barcodes

- RFID tags can have read/write memory capability, while barcodes do not

- Tags are less sensitive to undesirable environment

- Finding missing items are faster than when using the barcode

Applying this real-time RFID system is expected to benefit shoppers and supermarket management in the following aspects.

\subsection{Benefits to Shoppers}

Shopping store customers will be able to search for the exact location and find the detailed up-to-date information about products of different types.

Shoppers typically spend long time queuing to get through the checkout due to the following factors:

- Number of items being purchased: greater the number of items, the longer it will take to get through the checkout

- Time to unload cart

- Time that the cashier spend to scan the purchased items

- Time to pack the purchased items and finally

- Payment processing time

Queuing results in reducing satisfaction and elevating stress for shoppers.

By introducing RFID, it is possible to eliminate time required for scanning items and cart unloading process. RFID does not require scanning the purchased items. By adding a RFID reader to the cart, we will eliminate payment process. As the shopper adds or removes items to/from the cart, the RFID reader tracks these items and adds them to or removes from the purchaser's bill.

In total, as a result of using RFID, the customer shopping experience will be improved.

\subsection{Benefits to Organization}

- As a result of real time data collection, the supply chain companies have the latest information of their products status

- Agent processes collected data locally and only useful information is transmitted to the back-end application therefore increasing the performance of the system

- Increase in sales by using collected data on customer shopping behavior for marketing purposes

- Prevention of theft by activating the alarm (when someone tries to leave the supermarket without having the tag item disabled at the checkout)

- Efficient inventory management and notifying supermarket management that a certain product is running out of stock and needs to be restocked

- Reduction the cost of operation e.g., lower cost of goods sold which comes in the form of labor efficiency in areas of inventory management and checkout operation

- Improved ability to monitor and control of the supermarket services, for example monitoring the current temperature of frozen products 
The primary objective of this research is designing of a convenient shopping cart and thus of better shopping environment. In addressing these needs, we propose a framework architecture based on a distributed computing architecture that adopts intelligent agent technology with an RFID based application. RFID provides the capability to uniquely identify an object within a supermarket area, while agents are able to establish a channel of communication which can be used to provide connection between RFID devices and supermarket back-end system to achieve the above mentioned goals. The proposed framework includes a design of mobile shopping cart equipped with both RFID and agent technologies.

Integrating RFID and agent technologies is an effective way to process the continuous flood of RFID data. Agents collect real-time data received by RFID reader. This integration would allow goods with RFID tags to be checked out through supermarket gates that contain RFID readers.

The remainder of this study is organized as follows. Section 2 presents related work. Reviews the RFID and agent technologies given in section 3. Section 4 will introduce the overall framework architecture of the proposed agent based RFID supermarket information system. Section 5 provides final conclusions.

\subsection{Related Work}

A lot of efforts have been spent to develop standards, middleware and applications for the radio frequency identification systems. The industry has already made large investments into developing and implementing this technology. However, the RFID technology is still in its early stage. Because of this, middleware systems are expected to undergo frequent redesigns. The proposed an agent-based middleware for RFID systems situated between the reader and software application which uses the EPC data. The main objective of this RFID middleware is to process and manage data produced by RFID systems and send them to the computer systems. Such middleware controls the distribution of information to different business applications and handles large amount of data by filtering and aggregation of it.

Different middleware platforms are proposed by researchers: Moo open source RFID platform (Zhang et al., 2011), Win RFID (Prabhu et al., 2008), middeware based on group communications in peer-to-peer networks (Cervantes et al., 2007), Object-Oriented Distributed Middleware (Villanueva et al., 2012), Logistics Information Technology (Chen and Tsai,
2010), REFiLL (Anagnostopoulos et al., 2009). A survey by (Al-Jaroodi et al., 2009) was conducted that had compared these middleware platforms. There are other middleware platforms available which are developed by different vendors such as SUN, BEA Systems and IBM (2012). The most relevant are multiple agent based RFID middleware (Selamat and Lockman, 2009; Massawe et al., 2010; Rekleitis et al., 2010; Agarwal et al., 2011) where multiple agents are applied for verification and validation collected information. Boulaalam et al. (2011) proposed an architecture that is based on mobile agents and RFID for tracking and managing information of the whole product lifecycle in the extended enterprise.

Most of these systems do not consider the disrupting behavior of the crowd at checkouts in supermarkets. The proposed architecture focuses on solving the problem that happens when many RFID objects (within carts) are moving at the same time toward the supermarket checkpoints using multi-agent approach.

Previous research has addressed the integration of RFID technology with multi-agent approach (Massawe et al., 2010; Bade and Lamersdorf, 2011). Some of the works use a centralized approach to process and manage incoming RFID signals from RFID readers. This approach may lead to a single point of failure and performance problems. A typical RFID supermarket network can have large numbers of RFID readers attached to it. Handling data coming from these readers can present a challenge to the performance of the middleware. In order to handle such a challenge a distributed network technique is proposed in which each node is responsible of gathering data from its designated source of data.

\subsection{Rfid and Agent-Based Technology}

\subsubsection{RFID}

RFID tags can be classified into three major types based on their power source: active (battery-assisted and true active), passive no internal battery (powered by energy from reader) and semi-passive (Symonds and Ayoade, 2010). The active RFID covers greater range; it broadcasts a signal to a reader and thus requires less power from it. Passive RFIDs are smaller and lighter and obtain power from a reader. Semi-active tags are the same as active tags but their battery is used to power the onboard microchip and not broadcast a signal. The reader sends electromagnetic waves that induce current in the passive tag's antenna, the tag reflects the RF signal transmitted and adds information by modulating the reflected signal.

RFID supports three types of memory (Paret, 2005): 
- Read Only: Data cannot be modified after it's manufactured. Stores limited size of data usually 96 bits

- Read-write: Data can be read and write from/on tag dynamically. It can store up to 128 Kbytes. It's more expensive than the Read Only. These tags are more complex than read only tags

- Write-once/read-many: can be programmed only once

An active tag operates with up to $1 \mathrm{MB}$ of memory. Some tags are recyclable as they can be reprogrammed to store new data.

The tag is attached to or integrated with objects such as products, cases, or pallets. They can also be embedded in identification documents or devices. Tag attached to a product can be read by RFID reader which identifiesthe object from the information on the tag. In the most mentioned applications tags tend to be mobile and readers are stationary. RFID tags operate in three frequency ranges: low frequency (LF, 30 500 kHz), high frequency $(\mathrm{HF}, 10 \sim 15 \mathrm{MHz})$ and Ultra High Frequency (UHF, 860 960 MHz, $2.45 \mathrm{GHz}$ and $5.8 \mathrm{GHz}$ ) (Jechlitschek, 2006).

Among the existing mobile RFID technologies is the Near Field Communication (NFC). NFC is a short-range wireless technology which is operating in $13.56 \mathrm{MHz}$ frequency ranges and reading distance of $10 \mathrm{~cm}$. Usually NFC uses LF and HF frequency bands. Frequencies of LF and HF tags are license exempt and can be used anywhere.

The most widely used RFID tags are based on the UHF band of 860 960 MHz,2.45 GHz and 5.8 GHz. UHF tags have the highest transmission rate and range among all tags. Their range is from 3 to 6 meters for passive tags and more than 100 meters for active tags. Frequencies of UHF tags require authorization and are different from country to country. The UHF banded RFID tags are cheap and already are used in many RFID applications. Such a tag can be read from longer distances and faster than the LF (Low Frequency125-134 kHz) and HF (High Frequency13.56 MHz) banded RFID tags.

The typical architecture of RFID system defined by EPC global consists of RFID tags, which are embedded in or attached to an object, an RFID reader and back-end application as shown in Fig. 1.

\subsection{Components of the RFID Architecture}

\subsubsection{Reader}

Scans the tag attached to an object (or multiple tags simultaneously) and transmits tag information to a host computer, so that objects may be identified, located and tracked. The reader should be able to handle large number of signals being emitted from tags at the same time. There are two types of readers: stationary (the reader is on a fixed location) and mobile (the reader is integrated with a mobile device or supermarket cart). A reader has an interface unit enabling it to interact with intelligent devices. RFID readers are able to establish a channel of communication, read the tags and trace movement of these objects within the coverage area. Each reader is responsible for a particular zone. Multiple readers located in different places sending data through wired or wireless networks.

\subsection{Tag}

A memory device, usually EEPROM, programmed with a series of bits, supported by antenna to emit RFID signal. The tags can be used at only certain frequencies and have only certain read and write bits on the tag to secure the read-write operations.

\subsection{Back-end System}

It is responsible for managing all information related to the tagged items.

The RFID system is working in the following way: RFID tag readers activate RFID tags through radio frequency signals, tags' information is transmitted to the readers. Than readers send the information to the back-end system via wired or wireless network to be processed (Ping-Chuan and Guo-Feng, 2010). The back-end system contains information on the RFID tags attached to the items.

\subsection{Agent Technology}

An agent is an intelligent reusable executable software on a network which can carry out autonomous tasks or delegate the execution of these tasks to another agent or set of agents. Agents are capable to complete a predefined task autonomously and with minimal users' involvement. It has a degree of control over its actions and it does not always have to wait for commands. It adapts its behavior in response to the changing environment. A mobile agent has the property that its state information and code can be moved to another server within the same network and then continue execution from where it stopped on the previous server. The operation of agents is supported and managed by distributed agent platform systems. An agent can migrate from server to server in heterogeneous environments. Agents communicate with each other according to the organizational structure to accomplish specific tasks. 


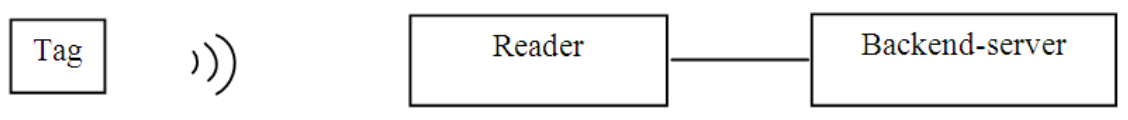

Fig. 1. RFID architecture

On the server side, the agent interacts with stationary services and other resources to accomplish its mission. It can communicate with other agents to anticipate, adopt and plan tasks. Its behavior consists of beliefs and desires. Agent's interactions depend on the location of an entity within an agent-based system.

Agent technology thus represents an excellent solution for solving problems of applications ranging from large distributed complex systems to small ones. The program of the mobile agent is small and its movement within the network will significantly reduce the usage of network bandwidth and the response time. When a mobile agent is dispatched there is no need to wait for it to return. The original connection to the network does not need to be maintained while the mobile agents are roaming through it. The mobile agents can wait until the original connection is reestablished on the network before attempting to return.

Software agents can be considered an attractive technology for implementation in supermarket information systems. Mobile agents are suitable for tracking product's information in supermarket environment and support the mobility aspect (at any time anywhere). Mobile agents can process data locally and migrate to supermarket back-end server. This approach can provide other benefits, such as reduction of network traffic, asynchronous interaction, filtering and so on. It also can handle huge heterogeneous collection of data generated by RFID readers in a poor network condition.

\subsection{Proposed Architecture of Agent Based Rfid}

\subsubsection{Architecture}

The proposed architecture consists of four main components, namely the RFID tags, RFID reader, RFID middleware and RFID applications. RFID middleware is located between readers and RFID applications. RFID reader detects tags attached to objects, the tags data are delivered to RFID middleware for processing and then are sent to back-end RFID applications as shown in Fig. 2. RFID reader can deal with multiple tags simultaneously. Reading multiple tags (items) in a supermarket environment is a key decision to be made in term of how many items need to be read in and in what time frame. Anti-collision algorithms should be implemented for the tags if several of them are to be read simultaneously as described in (Piramuthu, 2008).
These algorithms reduce overall read time and maximize the number of tags to be read at the same time. Multiple readers can be connected to the RFID middleware at the same time. The middleware software usually runs on server and provides an interface to RFID application, to achieve cross platform hardware integration. RFID middleware manages readers, filters and aggregates captured RFID data, ensures data accuracy, prevents data redundancy and delivers these data to the back-end applications (Manish and Moradpour, 2005). Tags data received from each connected reader are accepted by RFID middleware monitoring agent. This approach will increase the performance of the middleware, especially in case of heavy traffics to handles large volume of data, by generating a mobile agent for each reader request and all these agents are working at the same time.

RFID application performs business logic based on the collected data from the RFID middleware. The backend subsystem is responsible for managing all information related to the tags. It holds information on all tagged objects in close vicinity of a supermarket, for example products, carts, readers; application software that performs business specific functions; and a server agent as communication point with other agents within the proposed system.

The RFID middleware communicates and exchanges data with the server agent of the back-end subsystem by deploying mobile agent carrying collected data from tags. Interface agent communicates with the mobile agents to specify the data they wish to receive and the kind of data they want to be reported. Mobile agents in turn communicate with the appropriate reader agents, collect the raw data, process it, generate the requested reports and send them all back to the interface agent.

\subsection{Shopping Cart Technology}

The proposed shopping mobile cart is equipped with a RFID reader, a RFID antenna and an embedded computer system. A LCD touch screen is mounted at the front of the cart and acts as a user interface console that facilitates and monitors customer operations. When a product is dropped into the cart, the cart's RFID reader will be able to identify the profile of each product via its RFID and display product information (name and cost of the product, product specifications and features, consumer reviews) on a LCD touch screen. 


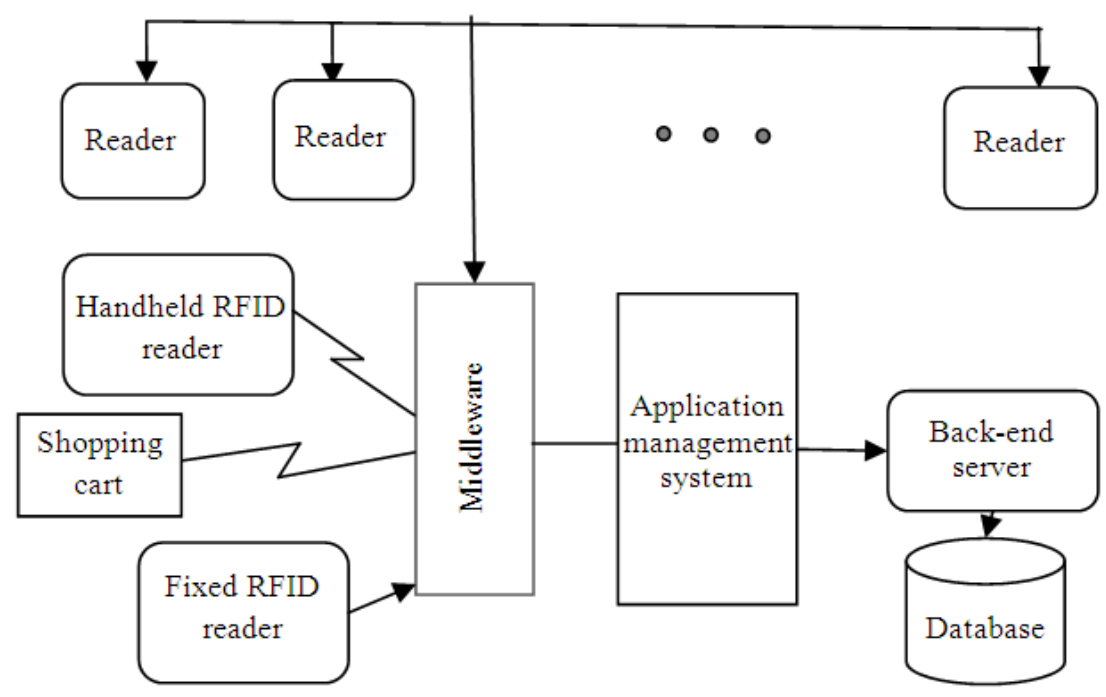

Fig. 2. Simple RFID system

The RFID signal is limited to within the shopping cart. It will carry data on items in the cart with their prices, as well as the total price. The store will also be able to play advertisements on the LCD based on the physical location of the customer in the supermarket. Such technology offers consumers access to more product information, the ability to find the location of the product and is reviewing the customer's accumulated items. At the check-out point, shopping cart system transmits the list of products and total cost to the cashier using wireless technology.

The cart's mobile agent communicates with the back-end RFID application via the wireless supermarket network. Using mobile agent in this design will reduce the power consumption since the size of the mobile agent software is small. The cart has two RFID devices: a product tag reader for items in the cart and a cart tag. We will refer to the supermarket product tags as product-tags to distinguish them from the cart-tags identifying carts in the supermarket. Whenever the customer is located near the final check-out point, RFID reader that is close to that area would read the data of the cart (saved on the cart tag), launch a mobile agent to the back-end system and return back with a list price of those items. The returned list is then compared with one generated by the cart mobile agent.

\subsection{Agents}

The following agents are used for the proposed architecture.

\subsubsection{Mobile Agent}

Mobile agent moves within the system that contains an object communicate. They can be dynamically generated during the execution and can reconfigure themselves dynamically based on changes of the services.

\subsection{Middleware Agents}

\subsubsection{Coordinating agent}

This agent will generate and send one or multiple mobile agents to the backend server. It's responsible for the connectivity to the supermarket databases. This agent facilitates interactions among and between agents.

\subsection{Information Agent}

It is dynamically created when changes in the environment (new RFID tags) are detected. The information is provided to this agent by RFID readers at check out points. This agent is used to support the process of shoppers not using shopping cart.

\subsection{Cart Agents}

\subsubsection{Cart Mobile Agent}

Is an autonomous, software entity that manages only RFID tags within the cart. It represents the interests of an in-store customer. It will carry the data of the checked out items from the cart RFID reader. When all purchases are made, the agent will move towards the back-end system though the middleware and returns to the user's 
location with the data after it has completed the transaction. Each mobile cart agent is responsible for a single cart containing a group of RFID tags.

\subsection{Cart User Interface Agent}

The user interface agent is a static agent that interacts with the user. It is expected to coordinate with cart mobile agent.

\subsection{Cart Monitoring Agent}

Within the cart there is a static monitoring agent to monitor new tags added to or removed from the cart.

\subsection{Back-End Server Agent}

It receives a request from mobile agent and search the supermarket database. The result passed to the mobile agent, who goes back to the web server and deliver the result to the coordinating agent of the middleware.

\subsection{Device Agent}

Each main supermarket device, such as a freezer, has an agent attached to it. Devices can communicate and cooperate with main system through their agents. When a device is turned on, the agent requests and receives the device operational information from the central system and can manipulate its device according to specific rules.

\subsection{Supermarket RFID-Based Mobile System}

The RFID mobile platform will help users carrying mobile RFID devices and staff holding mobile RFID reader to communicate with system via wireless network and to connect to the back-end system via a data exchange interface. A mobile phone with RFID reader can read tags and get the information on the tagged object anywhere within the supermarket because a mobile RFID reader can easily communicate with the back-end server via wireless communication network. The size of the tag code is small as defined by Electronic Product Code (EPC) global of length of 96 bits or 128 bits (PIA, 2011). This small code size makes the operations on tags very efficient in wireless environment. A mobile phone with built-in Near Field Communication RFID reader (or as a NFC RFID reader sticker attached to the back of it) allows customers to download information to their mobile phones against tags attached to store shelve items. When a customer taps the mobile phone against a tag, the mobile phone captures data about that item by linking to supermarket information system. Such customers can have access to various supermarket services. This RFID subsystem enables customers to instantly charge their purchases to their credit card. Customers carrying such devices place their mobile phone in front of an electronic reader at the checkout counter.

Customers using the RFID mobile system will find some shopping support services such as detailed information on tagged item, promotions, prices.

In recent years several vendors supply mobile RFID enabled readers to the market that can handle both NFC and RFID applications (McHugh and Yarmey, 2012) and the handheld type UHF RFID reader. These RFID readers have the ability of reading tag code wirelessly (Owunwanne and Goel, 2010). In addition to that there are a number of academic researches that propose a mobile RFID reader architecture which is implemented in cellular phone (McHugh and Yarmey, 2012; Lee et al., 2010).

\section{CONCLUSION}

The aim of this research is to apply the radio frequency identification and software agent technologies in the design of a supermarket information system, which will help to overcome the supermarket's problems by creating a simple and convenient shopping environment, as well as reducing operational costs and increasing productivity. Using the RFID can minimize human errors, increases flexibility, efficiency and save time and customers carrying RFID mobile devices can communicate with system using their mobile devices via wireless communication network and connect to the backend system via a data exchange interface.

The proposed system is supported by agent based RFID shopping cart. Such cart technology offers consumers access to more product information, such as the location of the product, advertisements on the screen of the cart based on the physical location of the customer in the supermarket.

\section{REFERENCES}

Agarwal, A.A., S.K. Sultania, G. Jaiswal and P. Jain, 2011. RFID based automatic shopping cart. Control Theory Inform., 1: 39-44.

Al-Jaroodi, J., J. Aziz and N. Mohamed, 2009. Middleware for RFID systems: An overview. Proceedings of the 33rd Annual IEEE International Computer Software and Applications Conference, Jul. 20-24, IEEE Xplore Press, Seattle, WA., pp: 154-159. DOI: 10.1109/COMPSAC.2009.129 
Anagnostopoulos, A.P., J.K. Soldatos and S.G. Michalakos, 2009. REFiLL: A lightweight programmable middleware platform for cost effective RFID application development. J. Pervasive Mobile Comput., 5: 49-63. DOI: 10.1016/j.pmcj.2008.08.004

Bade, D. and W. Lamersdorf, 2011. An agent-based event processing middleware for sensor networks and RFID systems. Comput. J., 54: 321-331. DOI: 10.1093/comjnl/bxp103

Bendavid, Y., S.F. Wamba and L.A. Lefebvre, 2006. Proof of concept of an RFID-enabled supply chain in a B2B e-commerce environment. Proceedings of the 8th International Conference on Electronic Commerce: The New E-Commerce: Innovations for Conquering Current Barriers, Obstacles and Limitations to Conducting Successful Business on the Internet, (SBI' 06), ACM Press, New York, USA., pp: 564-568. DOI: 10.1145/1151454.1151461

Boulaalam, A., E.H. Nfaoui and O.E.L. Beqqali, 2011. Mobile agent PLM architecture for extended enterprise. Int. J. Comput. Sci., 8: 55-61.

Cervantes, F.L., Y.S. Lee, H. Yang and J. Lee, 2007. A hybrid middleware for RFID-based parking management system using group communication in overlay networks. Proceedings of the International Conference on Intelligent Pervasive Computing, Oct. 11-13, IEEE Xplore Press, Jeju, pp: 521-526. DOI: $10.1109 /$ IPC.2007.112

Chen, C.Y. and P.W. Tsai, 2010. A design of multiheterogeneous system using SOA and RFID middleware platform. Proceedings of the International Conference Machine Learning and Cybernetics (MLC' 10), pp: 2956-2961.

GIAI, 2012. Radio Frequency Identification (RFID) technology-a global strategic business. Global Industry Analysts, Inc.

IBM, 2012. Websphere sensor events: Integrate sensor data to create new visibility and drive business process innovation.

Jechlitschek, C., 2006. A survey paper on Radio Frequency Identification (RFID) Trends.

Lee, D., S. Kim, H. Kim and N. Park, 2010. Mobile platform for networked RFID applications. Proceedings of the Seventh International Conference on Information Technology: New Generations, Apr. 12-14, IEEE Xplore Press, Las Vegas, NV., pp: 625630. DOI: $10.1109 /$ ITNG.2010.188
Luo, Z. and H. Wang, 2012. Research on intelligent supermarket architecture based on the internet of things technology. Proceedings of the 8th International Conference on Natural Computation, May 29-31, IEEE Xplore Press, Chongqing, pp: 1219-1223. DOI: 10.1109/ICNC.2012.6234594

Manish, B. and S. Moradpour, 2005. RFID Field Guide: Deploying Radio Frequency Identification Systems. 1st Edn., Prentice Hall PTR, Upper Saddle River, ISBN-10: 0131853554, pp: 263.

Massawe, L.V., J. Kinyua and F. Aghdasi, 2010. An implementation of a multi-agent based RFID middleware for asset management system using the JADE platform. Proceedigns of the IST-Africa, IEEE Xplore Press, Durban, May 19-21, pp: 1-8.

McHugh, S. and K. Yarmey, 2012. Near field communication: Introduction and implications. J. Web Librarianship, 6: 186-207. DOI: 10.1080/19322909.2012.700610

Owunwanne, D. and R. Goel, 2010. Radio Frequency Identification (RFID) technology: Gaining a competitive value through cloud computing. Int. J. Manage. Inform. Syst., 14: 157-164.

Paret, D., 2005. RFID and Contactless Smart Card Applications. 1st Edn., Wiley, Chichester, ISBN-10: 0470011955, pp: 330.

PIA, 2011. EPCglobal. Privacy Impact Assessment.

Ping-Chuan, Z. and M. Guo-Feng, 2010. Design of passive rfid reader based on mobile phone. Proceedings of the 2nd International Conference Networks Security Wireless Communications and Trusted Computing, Apr. 24-25, IEEE Xplore Press, pp: 446-449. Wuhan, Hubei, DOI: 10.1109/NSWCTC.2010.239

Piramuthu, S., 2008. Anti-collision algorithm for RFID tags. Proceedings of the Conference on Mobile and Pervasive Computing (MPC' 08), pp: 116-118.

Prabhu, B.S., X. Su, H. Ramamurthy, C.C. Chu and R. Gadh, 2008. WINRFID-a middleware for the enablement of Radio Frequency Identification (RFID) based Applications. UCLA-Wireless Internet for the Mobile Internet Consortium.

Rekleitis, E., P. Rizomiliotis and S. Gritzalis, 2010. An agent based back-end RFID tag management system. Proceedings of the 7th International Conference on Trust, Privacy and Security in Digital Business, (SDB' 10), Springer-Verlag Berlin, Heidelberg, pp: 165-176. 
Selamat, A. and M.T. Lockman, 2009. Multi-agent verification of RFID system. New Challen. Comput. Coll. Intell., 244: 255-268. DOI: 10.1007/978-3642-03958-4_22

Symonds, J. and J. Ayoade, 2010. The RFID tag pictorial glossary project. Proceedings of the IEEE International Symposium Technology and Society, Jun. 7-9, IEEE Xplore Press, Wollongong, NSW., pp: 190-195. DOI: 10.1109/ISTAS.2010.5514640

Takai, K. and K. Yada, 2010. Relation between staytime and purchase probability based on RFID Data in a Japanese supermarket. Proceedings of the 14th International Conference on KnowledgeBased and Intelligent Information and Engineering Systems, (IES' 10), Springer-Verlag Berlin, Heidelberg, pp: 254-263.
Villanueva, F.J., D. Villa, F. Moya, M.J. Santofimia and J.C. Lopez, 2012. Internet of things architecture for an RFID-based product tracking business model. Proceedings of the 6th International Conference on Innovative Mobile and Internet Services in Ubiquitous Computing, Jul. 4-6, IEEE Xplore Press, Palermo, pp: 811-816. DOI: 10.1109/IMIS.2012.59

Zhang, H., J. Gummeson, B. Ransford and K. Fu, 2011. Moo open source RFID platform. 